\title{
Women with breast cancer: Embracing and distancing themselves from the term cancer survivor
}

\author{
Wilson H. Stephenson ${ }^{1}$, Paula C. Fletcher ${ }^{2}$, Margaret A. Schneider ${ }^{2}$ \\ 1. Northern Ontario School of Medicine, Lakehead University, Cananda 2. Kinesiology and Physical Education, Wilfrid \\ Laurier University, Canada.
}

Correspondence: Paula Fletcher. Address: 75 University Avenue West, Waterloo, Ontario, Canada.

Email: pfletcher@wlu.ca

Received: August 18, 2013

DOI : $10.5430 /$ cns.v2n1p64

\author{
Accepted: November 27, 2013 \\ Online Published: December 22, 2013 \\ URL: http://dx.doi.org/10.5430/cns.v2n1p64
}

\begin{abstract}
The purpose of this study was to examine the lived experiences of women who had been diagnosed with breast cancer, specifically focusing on the meaning they associate with the term cancer survivor. A woman who has been diagnosed with breast cancer in North America undoubtedly will encounter the term cancer survivor at some point following her diagnosis. However, few studies have been focused on examining the meaning of this term for women who have been diagnosed with breast cancer, let alone whether these women embrace the use of this term. The lived experiences of nine women between the ages of 35 to 58 years were examined using in-depth, semi-structured interviews with interpretive phenomenology as a theoretical orientation. Data collection continued until three distinct themes emerged and saturation of data was achieved for each theme. The results of this study are focused on the meanings associated with the term cancer survivor for these women including identification, safety, and behavior change. A continuum model was developed from the data in order to illustrate the complexity of this subject matter. A person's position along the continuum depends on the meanings that person associates with the term cancer survivor (identification, safety, or behavior change) and that person's feelings, either positive or negative, towards those meanings. As such, this continuum demonstrates how a woman can embrace certain aspects of the term cancer survivor and distance herself from other aspects. These findings will benefit clinicians because attaining a rich description of a woman's lived experience with breast cancer will help them to understand and address psychosocial issues that arise throughout the breast cancer journey.
\end{abstract}

\section{Key words}

Breast cancer, Cancer survivor, Psychosocial oncology, Illness experience, Identity

\section{Introduction}

A diagnosis of cancer can result in an automatic association with the term cancer survivor ${ }^{[1]}$. This association may come as a surprise to some, as being diagnosed with cancer could be more readily associated with intense fear regarding the prospect of uncomfortable medical treatments and possibly even death ${ }^{[2]}$. However, the National Coalition for Cancer Survivorship (NCCS) ${ }^{[3]}$ states that every person who is diagnosed with cancer is considered a cancer survivor. This statement has started a thriving debate and has authors calling for a better understanding of this term ${ }^{[4-6]}$. Perhaps this debate is most poignant within the breast cancer culture in North America ${ }^{[5]}$ because breast cancer is the most common 
cancer among North American women ${ }^{[7,8]}$, receives the most funding ${ }^{[9]}$, and arguably is the most recognized malignancy ${ }^{[10]}$. As Bartels ${ }^{[1]}$ stated about the breast cancer culture "the vocabulary of survival is everywhere: on web-sites, in support groups, in innumerable publications for people who have come into contact with cancer, and in cancer stories".

Using the term cancer survivor instead of other terms, such as patient or victim, is meant to empower people diagnosed with cancer ${ }^{[11]}$, which has certainly been the case for some; the popularity of fundraising and advocacy events, such as the Susan G. Komen Run for the Cure, are an example of this ${ }^{[10]}$. In other cases, the term cancer survivor is rejected because it evokes specific images ${ }^{[12]}$. As one author put it: "[the culture of survivorship] works to legitimize particular forms of publicity and participation at the same time that it helps to marginalize others" ${ }^{[12]}$. Considering how pervasive the term cancer survivor is, it does not seem likely that this terminology will be removed from the cancer lexicon in the near future. However, by interpreting this term from differing perspectives more people who have been diagnosed with cancer may be able to use this term for its originally intended purpose - as a metaphor to find meaning in the cancer experience ${ }^{\text {[13-16] }}$.

Many authors have written about the culture of breast cancer in North America ${ }^{[1,17-24]}$. However, few studies have been designed to include women who have been diagnosed with breast cancer and analyze their perceptions of the breast cancer culture ${ }^{[25-30]}$. Even fewer studies have been focused on these women's feelings towards the term cancer survivor ${ }^{[12,31,32]}$. Of these three studies, Helgeson's ${ }^{[32]}$ work was quantitative and was not designed to ask women open-ended questions about the term cancer survivor, whereas Document et al.'s ${ }^{[31]}$ and Kaiser's ${ }^{[12]}$ works were qualitative in nature and specific questions were asked of the women about their perceptions toward this term.

The participants in Kaiser's ${ }^{[12]}$ study included 39 women with ages ranging from 28-87 years (mean $\left.=52\right)$ who had completed treatment for breast cancer three to 18 months prior to the interview date, although some were still receiving tamoxifen. In the analysis of in-depth, semi-structured interview transcripts Kaiser determined that the participants described a breast cancer survivor as a "triumphant, happy, healthy, and feminine" woman. While some of the participants of this study embraced this perspective the majority of participants rejected this dominant perception or made modifications to and subsequently attempted to distance themselves from it ${ }^{[12]}$.

Document et al. ${ }^{[31]}$ analyzed the responses to 83 open and closed telephone interview questions completed by women who had been diagnosed with breast cancer $(\mathrm{N}=112)$. Analysis revealed that almost all participants $(\mathrm{n}=107)$ considered themselves cancer survivors. The findings of this study conflicted with Kaiser's ${ }^{[12]}$ statement that a majority of participants did not embrace what she deemed to be the culturally dominant cancer survivor identity. The difference in recruitment methods may account for the discrepancy found between these two studies. Document et al. ${ }^{[1]}$ recruited all participants from either a Susan G. Komen event or a Celebration of Life Luncheon, groups which are likely to foster the cancer survivor identity. Kaiser ${ }^{[12]}$, on the other hand, recruited participants through a cancer support centre and personal referrals, which may be less likely than the previously mentioned organizations to foster the cancer survivor identity. Support for this explanation comes from a study conducted by Morris et al. ${ }^{[33]}$ who studied the results of a 1,000 mile motorcycle ride with women who had been diagnosed with breast cancer. They observed that before the women went on the ride together, many did not consider themselves survivors. After the ride, many of the women reported that they felt they were survivors. The authors attributed this change to belonging to a group.

The aim of the current study was to contribute to the ongoing debate about the meaning of the term cancer survivor to women who have been diagnosed with breast cancer. We examined the extent that women who have been diagnosed with breast cancer accepted or rejected the cancer survivor identity. The typical characteristics of the cancer survivor identity were reported by participants in the study and their acceptance or rejection of those characteristics was observed. 


\section{Methods}

\subsection{Theoretical orientation}

Interpretive phenomenology ${ }^{[34]}$ was used as the guiding theoretical orientation for this study. According to van Manen, a phenomenological analysis is a "bringing to speech of something" ${ }^{[34]}$ and provides a fuller and deeper interpretation of that something in order to evoke within its audience a recognition of freedom within human experience.

\subsection{Participant recruitment}

Upon receiving ethics approval from the Wilfrid Laurier University Research Ethics Board, participants (N=9) were recruited using a purposeful sampling technique via a recruitment poster. The purposeful criteria included that participants must: 1) be female, 2) be between 30 and 60 years of age, 3) have been diagnosed and treated for a form of breast cancer by a medical doctor, and 4) have completed primary treatment a minimum of one year and no more than ten years prior to the interview. The final criterion was selected so that participants would have at least one year since finishing treatment to experience life after breast cancer. A cut-off of ten years was selected so that the breast cancer experience would likely still be vivid in the participants' memories. The recruitment poster was distributed through a support group and key informant located in a rural Northern Ontario city. Five participants were recruited from the support group and three participants were recruited by the key informant. Additionally, one participant was recruited via snowball sampling, which is a sampling method where word-of-mouth is used to recruit new participants ${ }^{[35]}$.

\subsection{Data collection}

Four data collection tools were used: 1) background questionnaire, 2) semi-structured interview, 3) field notes, and 4) member check. The background questionnaire included information such as date of birth, marital status, highest level of education attained, diagnosis, age at diagnosis, cancer treatment received, and other questions related to the cancer experience with an emphasis on the participants' perspectives related to the term cancer survivor. The background questionnaire was designed to set a context for the interview and the information from the background questionnaire allowed the principle investigator (PI) to prepare for each interview in order to obtain the most information rich data possible.

Digital recording of all interviews ensured accurate documentation of participants' statements for the purposes of transcription following the interview. All interviews were conducted and transcribed by the PI (WS). The average interview length was 88 minutes (range $=50$ to 114 minutes). The interview was semi-structured in nature and contained a number of questions and probes that were used to clarify or elaborate upon participant responses to the initial questions. The questions were designed in order to create a balance between the potential negative and positive aspects of the breast cancer experience and to elicit detailed information regarding acceptance or rejection of the cancer survivor identity (see Table 1).

Field notes also were used as an additional data collection tool. Field notes can be valuable in a number of ways ${ }^{[36]}$. First, they can help researchers remember what has occurred during their fieldwork. Second, they aid in the organization of observations made during field work. Third, they can contribute to data analysis processes because the creation of field notes based on observations require that the researcher has decided that specific observations are important to the study and thus the researcher has started to analyze the data ${ }^{[36]}$. Field notes were taken before, during, and after interviews with participants, as well as throughout the concurrent data collection and analysis component of the study (as explained in the data analysis section).

The final data collection tool employed was a member check ${ }^{[37]}$. In the current study, all participants received an e-mail or telephone call (depending on their preference) inviting them to review a copy of their interview manuscript. Six participants requested to receive their interview transcripts, which were either e-mailed or printed and delivered to their 
preferred address, and three participants did not respond to the e-mail regarding their member checks. Five of the six participants that requested their transcripts returned their member checks with no changes or minor changes to grammar and spelling. One participant requested her transcript but did not return her member check.

Table 1. Interview questions

\begin{tabular}{|c|c|}
\hline Question Number & Question \\
\hline 1 & Could you please describe your current state of health? \\
\hline 2 & Could you please tell me about the time in your life when you were diagnosed with breast cancer? \\
\hline 3 & Could you tell me about the treatments you received for breast cancer? \\
\hline 4 & $\begin{array}{l}\text { You indicated on your background questionnaire that " } \mathrm{X} \text { ” has been a big part of your support network. } \\
\text { Could you tell me more about that? }\end{array}$ \\
\hline 5 & $\begin{array}{l}\text { In your background questionnaire you stated that you think about breast cancer } \\
\text { (weekly/monthly/yearly/never). Could you tell me more about that? }\end{array}$ \\
\hline 6 & $\begin{array}{l}\text { I would like to show you a number of terms related to being diagnosed with cancer. I would like you to } \\
\text { look at these terms and tell me what they mean to you. }\end{array}$ \\
\hline 7 & $\begin{array}{l}\text { Often when someone is diagnosed with breast cancer others will offer them advice. Do you have any } \\
\text { stories of people offering you advice about how to manage your experiences with breast cancer? }\end{array}$ \\
\hline 8 & $\begin{array}{l}\text { I would like to show you a quote from a woman who had been diagnosed with cancer: "As time went } \\
\text { on and I talked to friends and relatives about my [cancer], I noticed they had their own ways of making } \\
\text { sense of cancer and that these were often quite different from, or even opposed, to mine" }{ }^{[24]} \text {. What } \\
\text { does this quote mean to you? }\end{array}$ \\
\hline 9 & Do you think being diagnosed with breast cancer has resulted in any positive outcomes? \\
\hline 10 & Do you think being diagnosed with breast cancer has resulted in any negative outcomes? \\
\hline
\end{tabular}

\subsection{Data analysis}

Central to the process of analyzing data from a phenomenological perspective is the concept of Époche. The goal of Époche is to understand how the researcher's bias has influenced the data and to find ways to minimize this bias in order for the data to be represented in a form that can be trusted by those who read the research report ${ }^{[38]}$. In this manner, Époche is very similar to reflexivity ${ }^{[35]}$, which, in the context of qualitative research, is the knowledge of one's self. "To be reflexive, then, is to undertake an ongoing examination of what I know, and how I know it" ${ }^{\text {[35] }}$

The PI has two sources of bias with respect to the topic at hand. First is the PI's personal experiences with cancer; the PI was diagnosed with cancer at 11 years of age, and the PI's mother was diagnosed with breast cancer approximately four years previous to the initiation of the study. These prior experiences with cancer have shaped the PI's perspective regarding lived experiences with cancer. The PI's second source of bias is the literature review that was conducted prior to interactions with the participants. This literature review contained many conceptualizations of breast cancer, including the term cancer survivor, and likely influenced the PI's thoughts about these concepts.

A reflexive journal was kept throughout the entirety of the research project as an attempt to attain Époche . Patton's ${ }^{\text {[35] }}$ explanation of reflexivity guided the creation of the PI's reflexive journal. Specifically, while making entries in the reflexive journal the PI asked himself the two questions Patton suggested regarding reflexivity, namely "what do I know, and how do I know it" ${ }^{[35]}$. These questions were used to track the source of information (previous personal experiences or lived experiences of participants) available to the PI for analysis.

\subsection{Thematic analysis}

Following the verbatim transcription of each interview, the data contained within the interview were analyzed consistent with the ethos of interpretive phenomenology ${ }^{[34]}$. This study used the "selective or highlighting approach" ${ }^{[34]}$. This 
approach to thematic analysis requires the analyst to identify parts of the interview transcript that represent the ethos of the participant's lived experiences with the phenomenon. The analyst then interprets the meaning of the representative sections of the interview transcript to identify themes related to the phenomenon being studied ${ }^{[34]}$. Participant recruitment and interviews continued until saturation ${ }^{[39]}$ of the identified themes was reached.

\subsection{Credibility}

Methodological triangulation and investigator triangulation were employed in order to increase the credibility of the analysis ${ }^{[35]}$. As mentioned, this study employed four data collection methods: background questionnaire, semi-structured interview, field notes, and member check in order to ensure the credibility of the findings via methodological triangulation. With respect to investigator triangulation ${ }^{[35]}$, the PI and the two supervising professors were involved in data analysis. The analysis of each interview was completed by the PI following the analytical technique described above. Following the PI's analysis, the supervising professors (PF and MS) performed an expert audit review. An expert audit review involves the critical appraisal of the original analysis by researchers who are considered experts in qualitative methodology ${ }^{[35]}$. The supervising professors have completed extensive work in the field of qualitative research and were able to provide critical appraisals of the data analysis.

Table 2. Participant demographics

\begin{tabular}{|c|c|c|c|c|c|c|}
\hline Participant & $\begin{array}{l}\text { Age } \\
\text { (years) }\end{array}$ & $\begin{array}{l}\text { Type of } \\
\text { breast } \\
\text { cancer }\end{array}$ & $\begin{array}{l}\text { Years since } \\
\text { diagnosis }\end{array}$ & $\begin{array}{l}\text { Treatments } \\
\text { received }\end{array}$ & Work & $\begin{array}{l}\text { Married with } \\
\text { children? } \\
(\mathrm{Y} / \mathrm{N})\end{array}$ \\
\hline Amelia & 54 & IDC & 5 & Bilateral mastectomy & Nurse & Yes \\
\hline Bethany & 50 & DCIS & 1.5 & $\begin{array}{l}\text { Lumpectomy and } \\
\text { radiation therapy }\end{array}$ & Nurse & Yes \\
\hline Chloe & 35 & DCIS & 5 & $\begin{array}{l}\text { Mastectomy and } \\
\text { primary chemotherapy }\end{array}$ & Administrator & Yes \\
\hline Dawn & 51 & DCIS & 5.5 & $\begin{array}{l}\text { Bilateral Mastectomy, } \\
\text { primary and adjuvant } \\
\text { chemotherapy, } \\
\text { radiation therapy }\end{array}$ & Dietary Aid & Yes \\
\hline Ella & 45 & IDC & 3 & $\begin{array}{l}\text { Lumpectomy, } \\
\text { mastectomy, radiation } \\
\text { therapy, primary and } \\
\text { adjuvant } \\
\text { chemotherapy }\end{array}$ & Nurse & Yes \\
\hline Fiona & 54 & DCIS & 1.75 & $\begin{array}{l}\text { Lumpectomy, primary } \\
\text { chemotherapy, } \\
\text { radiation therapy }\end{array}$ & Secretary & Yes \\
\hline Grace & 54 & IDC & 4 & $\begin{array}{l}\text { Lumpectomy, primary } \\
\text { and adjuvant } \\
\text { chemotherapy, } \\
\text { radiation therapy }\end{array}$ & $\begin{array}{l}\text { Elementary } \\
\text { School Teacher }\end{array}$ & Yes \\
\hline Heather & 53 & $\begin{array}{l}\text { Not } \\
\text { Available }\end{array}$ & 3.75 & $\begin{array}{l}\text { Lumpectomy, primary } \\
\text { chemotherapy, and } \\
\text { radiation therapy }\end{array}$ & Social Worker & No \\
\hline Isabella & 58 & $\begin{array}{l}\text { Not } \\
\text { Available }\end{array}$ & N/A & $\begin{array}{l}\text { Mastectomy, primary } \\
\text { chemotherapy }\end{array}$ & Unemployed & Yes \\
\hline
\end{tabular}

Table 2 provides demographic information for the participants of this study. The names given are pseudonyms. Age and time since diagnosis for each participant was calculated at the date of the interview. Primary chemotherapy was differentiated from adjuvant chemotherapy based on the type of chemotherapy received (i.e. tamoxifen and aromatase inhibitors were considered adjuvant chemotherapy). All participants had completed primary treatment at least one year previous to the date of the interview. However, some participants were still receiving forms of adjuvant chemotherapy. Note. IDC stands for invasive ductal carcinoma, DCIS stands for ductal carcinoma in situ. 


\section{Results}

\subsection{Participants}

Nine women living in Northern Ontario, ranging in age from 35 to 58 years, were recruited for this study. All of the participants were diagnosed with a form of breast cancer by a medical doctor at least one and half years and no more than five and half years previous to being enrolled in the study. All of the participants were assigned a pseudonym to ensure their anonymity. Further, demographic information about participants is limited so as to not identify them. See Table 2 for more information.

All participants were able to give detailed descriptions of their current and past experiences related to breast cancer. During the concurrent data collection and data analysis process a number of repetitive themes emerged. The participants provided ample evidence to support the emerging themes and the study team determined that saturation of data for these themes had been reached. Participant recruitment was concluded at nine participants when we determined that the themes had reached saturation. Saturation of data occurs when the research team deems that subsequent interviews with new participants provides redundant information ${ }^{[35]}$.

Analysis of the participant interviews led to the identification of three themes related to the meaning of the term cancer survivor: 1) cancer survivor means identification, 2) cancer survivor means safety, and 3) cancer survivor means behavior change. Each theme was organized into a continuum to illustrate that there was a range in participant responses within each theme. All participant responses fell within the boundaries of the continuums. However, some responses were closer to one end of the continuum than the other. Further, many of the participants provided responses that fell on different places along the continuum. This means that responses from one participant were not all necessarily grouped to one end of the continuum and could hypothetically support the full range of responses for a given continuum. Finally, the themes were combined to form a larger continuum - "The Cancer Survivor Continuum.” The Cancer Survivor Continuum represents the different meanings associated with the term cancer survivor and whether an individual participant embraced the term and its meanings or attempted to distance herself from the term and its meanings. Figure 1 provides a visual representation of the continuum for each theme.

\subsection{Cancer survivor means identification}

When Dawn was asked "What does it mean to be a survivor?” She responded by saying "I'm alive." When Chloe was asked what term she used to describe herself with respect to cancer she said "cancer survivor, obviously - I'm five years clean.” Fiona and Grace respectively added conditions to the cancer survivor concept such as "and you have to go through your treatments... and now I'm a survivor," and "Of course I am a cancer survivor... So, uhm, survivor, you think of surviving something that you know could be deadly.” These quotes suggest that, to the participants, the term cancer survivor represented a person who had been diagnosed with cancer and remained alive. The quotes from Chloe, Fiona, and Grace also suggest that people may add conditions to the term cancer survivor such as: being cancer-free for an extended period of time, completing treatment, and outliving something that could have been deadly.

Used in this context, the term cancer survivor became the default term for a person living beyond a cancer diagnosis. The term is succinct (compared to the more cumbersome "person who had cancer") and easily recognizable by others. Used in this way the term cancer survivor was used as a reference tool. This could be equated to a person using the term Kleenex ${ }^{\circledR}$ to identify a facial tissue or Tylenol ${ }^{\circledR}$ to indicate that a person is referring to acetaminophen.

While some participants of this study embraced the term cancer survivor, others attempted to distance themselves from it because they desired some level of privacy with respect to their cancer diagnoses. Two ways participants attempted to do this were firstly being cautious about disclosing their breast cancer histories and secondly taking steps to hide visual cues of their breast cancer histories. 
The first way participants attempted to conceal their cancer histories was by being cautious about revealing this information to others. Bethany, for example, stated:

Like I said I'm kind of a private person about it. If I had to tell you I had breast cancer it's probably not happening... And that is because I just want [people] to treat me like they always did. So if they thought I was a pain in the neck and they didn't like me before I didn't want it to change because I got a diagnosis.

This quote from Bethany illustrated that she wanted to maintain a private illness experience in order to avoid special treatment from others and, perhaps, because she wanted to earn the affections of others as opposed to having them given to her "because [she] got a diagnosis." In a very similar manner Dawn stated: "[one of the negatives of breast cancer] is the way some people treat you - it took me getting cancer for my mother-in-law to like me.” This comment from Dawn indicated that she would have preferred if her mother-in-law accepted her for who she was as opposed to having accepted her as a result of her cancer diagnosis. One interpretation of the desire to maintain a more private illness experience is that some of the participants wanted to mitigate the attention their cancer experiences received from others. A quote from Heather helps to conclude this analysis. Heather explained the reason she does not use the term cancer survivor is because: "it's the loss of me in there, right, that cancer becomes you. And that's what people see... they don't see me first.”

Hiding visual cues served as the second way that participants attempted to conceal their cancer histories. As indicated in Table 2, every participant in this study received treatments that were accompanied by alterations in appearance (e.g. mastectomy resulting in the loss of (a) breast(s), chemotherapy resulting in the loss of hair, and the appearance of physical fatigue from treatments). The participants offered stories about how their bodies, to borrow a word from Amelia, caused increased "curiosity" from others. These stories were often highlighted by issues related to body-image but also included hints of the desire to maintain a more private illness experience than would be allowed if all the visual cues were left unattended.

Amelia explained that her decision to have reconstructive surgery was based partly on her perception of people's reactions to her body. She told a story about how she and her husband would notice people staring at her when they were in public places. These comments suggested that Amelia believed the appearance of her body was signaling to others that there was something different about her. Perhaps Amelia perceived that the general public recognized that she had breast cancer. Amelia concluded this story by saying "I think I [had reconstructive surgery] to not get as many stares, and to make me look more normal." She then added "But [the extra attention] didn't really bother me that much. Uhm, well that doesn't even make sense does it? Because I did it.” This story from Amelia highlights the fact that she desired to conceal her breast cancer history from the general public in order to maintain a more private cancer experience. Interestingly, Amelia reported that she does not shy away from her cancer experiences in other domains and potentially this accounts for the apparent self-contradiction she made at the end of her story. Amelia was involved in breast cancer related activities at work, in her recreational time, with her family and friends, and was a regular contributor to a breast cancer support group - to use her words "[breast cancer] consumes me, but it consumes me in a good way, not in a way to be fearful of it." One interpretation of Amelia's desire to conceal her breast cancer experiences from the public could be that she had no control over others' opinions of her experiences, and maintaining a private cancer experience meant that she could control, at least to some degree, what other people thought about her illness experience.

Fiona also made a very interesting comment related to the culture that surrounds breast cancer and her resistance to the identity associated with being a cancer survivor. At one point in her interview Fiona talked about the emphasis on advertising in the breast cancer culture and how the pink ribbon, which she alluded to as being the symbol of the breast cancer culture seemed to be everywhere. While elaborating on this point Fiona stated:

I've got gum in my car right now that has a breast cancer symbol on each piece of gum. I bought it because it was bubble gum mint! And when we were in the car my daughter goes look mom it's got the breast cancer ribbon! And I go "oh come on!” So I think that part it kind of bothers me. 
Fiona's resistance to the symbol of breast cancer is a sign of her resistance to typical cancer survivor identity. This becomes more obvious when Fiona's comment is contrasted against Dawn's comments regarding the pink ribbon. After the formal part of Dawn's interview had concluded she showed the interviewer around her house. Here is a snippet of the conversation that ensued:

Dawn: And here are all my pink ribbons (points to three pink ribbons on the fridge) and I have a china cabinet that's all full of [breast cancer related items] that I buy.

PI: Uhm, so what do those symbols represent to you?

Dawn: Breast cancer

PI: Mmhmm

Dawn: And surviving

The contrast between Dawn's embrace of the pink ribbon as a positive symbol of survivorship and Fiona's attempt to distance herself from this symbol is obvious. For Fiona the cancer survivor identity comes with certain associations many of which she does not embrace.

The participant responses that have been presented thus far indicate that each participant falls somewhere between identifying herself as a cancer survivor or desiring a more private illness experience. However, the continuum is not so simple. This is because many participants provided responses that would place them on a number of different points along the continuum. For example, within this theme it was reported that Bethany was actively involved in advocating for breast health awareness to her colleagues, a quote that would place her closer to the identification end of the continuum. However, Bethany also stated during her interview that she was cautious of disclosing her breast cancer history, a quote that would place her closer to the privacy end of the continuum. Similar arguments could be made for many of the other participants as well. This illustrates the fluidity and complexity of identifying with the term cancer survivor.

\subsection{Cancer survivor means safety}

The second theme illustrates a continuum between the participants' hopes that they were "cancer-free" and their fears that they would never truly be free from cancer. In this context, the term cancer-free conveys the participants' beliefs that they were free from the long-term threats that cancer could impose upon them. The cancer-free side of the continuum describes the participants' willingness to embrace the cancer survivor concept because it represented a diminished threat from cancer. Many participants provided responses that placed them closer to this side of the continuum than the other. For example, Grace stated:

As soon as I knew that I was stage one I thought I could actually call myself a cancer survivor because I know: I'm early, I know [it hasn't] spread throughout my body, and I know there's treatment. So yes I can call myself a survivor.

This quote illustrates that Grace used the term cancer survivor once she felt confident that she would not die as a result of her breast cancer diagnosis. Very similarly, Dawn added "Cancer survivor, that's me...I beat it and I don't really, well I don't really worry about it coming back, if it does, I'll kick ass again... I won the fight, and I'm gonna continue to win.” Clearly, Dawn believed that the threat of cancer was diminished. This belief was grounded in her association with the term cancer survivor and the qualities that she believed it represented: positive attitude, fighter, and winner, among others.

Second, the cancer survivor concept represented a completion of the most difficult parts of cancer treatment. To Chloe, being a cancer survivor meant that she was no longer subjected to the difficulties related to her cancer diagnosis and treatment: "Cancer survivor sticks out that I've survived everything that I have gone through, the treatments, the surgeries, 
the pain.” Ella and Fiona respectively added: "I do feel like I'm past the hard part," and "I feel a survivor and I feel it's so much behind me." All three of these quotes could be interpreted as evidence that some of the participants embraced the cancer survivor concept because, to them, it represented refuge from the immediate threats of death and cancer treatment.

Many participants provided responses that suggested it was unrealistic to think that they were completely free from cancer and, as such, participants closer to the left-hand side of this theme created a distance between themselves and the cancer survivor concept. Every participant in the study articulated that she feared the possibility that long-term effects could develop in the future. For some participants in the study, being cognizant of these possibilities created distance between themselves and the cancer survivor concept. For example, Heather stated:

I guess part of my thing is survivor, I don't know whether I have it, it could still be in me, so that's the other part that it doesn't, like that's not true, I can't say that... So I don't know, like it is, survivor should fit for a, you know a hurricane or something, not a disease... And you know, any doctor that I did talk to didn't stamp cured, right.

In this quote, Heather expressed her fear that cancer could not only recur but that she had no way of knowing if she was ever cancer-free.

Other participants alluded to ideas similar to those captured by Heather's comment. Bethany stated "I don't know if I'm ready to say the word survivor, I've used it the odd time but when I do, I go 'well, you know, you kinda got a little more time...' So sometimes I don't use it," which suggested that she was not completely confident that she was cancer-free because not enough time had lapsed since her treatment ended. In contrast to Bethany, Chloe stated: "I could always have something growing in me. It's a fear that I have every day, whether it's going to come back. I think I'm more scared now... because of the latency period.” The respective comments from Heather, Bethany, and Chloe in this section highlight that their fears of recurrence persisted for a long period of time following their breast cancer diagnoses.

A quote from Amelia serves as a conclusion for the analysis of the participants' hope that their breast cancers have been resolved and the fear that cancer may come back. Amelia stated: "Have I beaten this? Nobody knows if I beat it, I believe I have but I'm also a realist that it may come back. It may come back somewhere else and then I'll be back to [being a cancer patient] when it does." This quote suggests that although the participant believed she was a cancer survivor, she also recognized there was a realistic possibility her cancer could recur. This fear could have potentially served as a motivational tool for the behavior changes Amelia developed since being diagnosed with cancer (see Theme 3: cancer survivor means behavior change).

Within the context of this theme, cancer-free could also refer to the long-term impact that cancer can impose upon a person. As Dawn stated “just because it's over, doesn't mean it's over, you know?” Indeed, many of the participants of this study discussed how their breast cancer diagnoses continued to affect them daily long after their treatments had ended. When Fiona was asked by the PI "How often would you say you're reminded of cancer?" she responded by saying "every day" then gave a short derisive noise, raised her right arm, which was swollen as a result of lymphedema, and said "reminded?" as if to say that it is impossible to forget. In a similar manner Isabella stated: "I don't tell everybody now that I have breast cancer; I had breast cancer." It is interesting that Isabella corrected herself to say that she had breast cancer as opposed to having breast cancer at the time of the study. It is possible that this is just a slip of the tongue. However, it is also possible that Isabella's comment was related to the blurred lines that differentiate these concepts. On the one hand, Isabella was no longer considered in primary treatment for cancer (i.e. she completed surgery and chemotherapy). On the other hand, throughout her interview Isabella discussed a number of health issues she was managing at the time of the study that were related to her breast cancer experience (e.g. numbness in her foot, being forced to wear a prosthesis, etc.). As Heather stated earlier, the term survivor would be better suited to a storm as opposed to a disease. This comment is likely meant to convey that a storm has a distinct beginning and end whereas a person's experiences with cancer are ongoing. 
The quotes presented to support this theme outline the spectrum of the continuum between the participants believing they were cancer-free and the participants fearing that they were not free from cancer. However, similar to the cancer survivor means identification theme, responses from a single participant often illustrated the full range of the continuum. For example, early in her interview Fiona stated that she felt her cancer experience was behind her. However, later in the interview Fiona stated that she did not have to be reminded of her breast cancer experience because the lymphedema in her arm was a constant reminder.

\subsection{Cancer survivor means behavior change}

Theme three presents a continuum that ranges between embracing the cancer survivor concept because it leads to individual behavior changes and distancing oneself from the cancer survivor concept because it places too much emphasis on individual change. Participants reported that individual behavior changes were believed to be an important part of living a healthy lifestyle and avoiding subsequent negative experiences related to cancer. In this context the term behavior change refers to the reported behavior changes the participants adopted following the completion of their primary treatment. The participants of the study made many references to behavior changes they developed as a result of their desires to remain survivors. For example, Amelia said:

I'm going to do everything in my power to maintain that survivor, I used to be a red head for over 20, 25 years. I dyed my hair. And when I did the research and found out that the hair dye causes bladder cancer I stopped dying my hair, you know. When I realized that there's a higher incidence of breast cancer for women that have a bigger abdomen, I started losing weight, and then I realized that through running I could keep my weight stable. Well that's good for a cancer survivor.

The behavior changes that this participant undertook following her breast cancer diagnosis were motivated by her desire to remain a cancer survivor. Other participants, such as Dawn, responded in a similar manner: "[cancer survivor means] you've gotta change your ways. I used to be a smoker; I never did drink... I go to the health food store now, I try to eat better than I did.” To Dawn, the cancer survivor concept was more than a label that indicated her membership to a group of people who have been diagnosed with cancer (theme one) - cancer survivor indicated a particular lifestyle. Additionally, Grace stated:

I'm hoping [having a positive attitude] helped me... become a survivor also because I also think if I was down and depressed and with that, again comes the immune system, and not going to get well. I don't want to have a recurrence so maybe this may help me, I'm doing everything I can to not have one.

This response from Grace extends the cancer survivor concept into the realm of psychology. Specifically, Grace's comment indicated that, to her, being a cancer survivor was about maintaining a positive attitude in order to keep her immune system operating at maximum capacity.

The behavioral and psychological processes undertaken by the participants were motivated by the desire to reduce the threat of cancer and maintain their statuses as survivors. Becoming an advocate for health was another behavior change undertaken by the participants that embraced the cancer survivor identity. For example, five of the nine participants reported that they belong to a breast cancer support group. These participants often reported that they continued to attend support group meetings in order to benefit others. As Ella stated: "it's more for support for other people. You have new people coming into your [support] group and they always have lots of questions and it's more for support for them.”

Other participants discussed how they became health advocates in their day-to-day activities. Bethany told a story about discussing breast cancer screening with her co-workers. Grace echoed this story when she stated that "there's been a few incidences of close calls [related to cancer] on my staff where they turn to me for advice so I think it's important to give 
[support] back.” The fact that health advocacy became integrated into the participants' everyday lives is evidence that the cancer survivor identity has become an important component of who they are.

Participants also reported that they had an aversion to the behavior changes they thought to be associated with the cancer survivor identity. For example, following a discussion centered on activities arranged for cancer patients while living in a hospital setting (e.g. caroling, country karaoke, socials, knitting class, etc.). Heather had this to say "that is where I felt a lot like you get cookie cutter cancer patient... Sorry, there's many individual people here who aren't the cookie cutter type but it felt like you gotta be like this." Heather further elaborated by saying that the activities did not bother her but the positive atmosphere and the assumed participation in that positivity did. This quotation shows that Heather resisted the behavior changes she believed were expected of her because she was a cancer patient. Cancer advocacy groups, such as the NCCS, state that from the moment of diagnosis a person is a cancer survivor, which would mean that Heather's resistance to the expected behavior changes as a cancer patient were also resistance to cancer survivor identity.

\subsection{Summary of results}

The analysis of nine women's lived experiences with breast cancer and uses of the term cancer survivor resulted in the formation of three themes. These themes all included a range of responses to support or refute the key word for which they are named. For example, the first theme, Cancer survivor means identification, dealt with participants' comments regarding the sense of identity that is associated with the term cancer survivor. The continuum within this theme ranged from public identification at one end to privacy at the other. The remaining two themes were organized in a similar manner.

The fact that all nine participants provided support for most, if not all, of the themes suggests that it is very likely that the cancer survivor concept is not an all-or-none categorization (i.e. either a person is a cancer survivor, or the person is not). It is likely that a person will embrace certain meanings associated with the cancer survivor concept while distancing themselves from others. There were numerous quotes presented in these themes that illustrated how the participants supported a meaning associated with the term cancer survivor in one context but distanced themselves from that meaning in another context. As a result, it is more suitable to represent the extent to which a person embraces the cancer survivor concept using a continuum. Figure 1 represents the Cancer Survivor Continuum as a dynamic concept that is composed of the continuums contained within each of the three themes. The context a person is placed in and how that context affects the way the person perceives the meanings associated with the cancer survivor concept ultimately determines where they fall on the Cancer Survivor Continuum.

The Cancer Survivor Continuum is fluid and a person can be at any point along the continuum and that position can change over time. The results of this study illustrated the extreme boundaries of the continuum in order to convey the range of participant responses to interview questions. However, an examination of one participant's entire collection of responses to the term cancer survivor often resulted in her being placed somewhere in the middle of the continuum. Fiona's responses are a good example of this. In her interview, Fiona twice stated her affiliation with the term cancer survivor: "I'm a survivor" and "I feel [like] a survivor." Without any further examination these two quotes would likely lead a person to believe that Fiona embraces the term cancer survivor and the meanings associated with it. However, Fiona also provided comments in her interview that would suggest she shied away from her status as a cancer survivor. One interpretation of Fiona's responses towards the term cancer survivor and the meanings associated with it could be that she privately embraced the cancer survivor concept but because of her desire to maintain confidentiality she could not publically embrace it. Another interpretation could be that although Fiona embraced certain aspects of the term cancer survivor, such as the connotation of safety that the term implies, but she distanced herself from other meanings. This is most evident in her desire to suppress her cancer experiences so she can "have fun... and live [her] life without thinking about [cancer] all the time." 


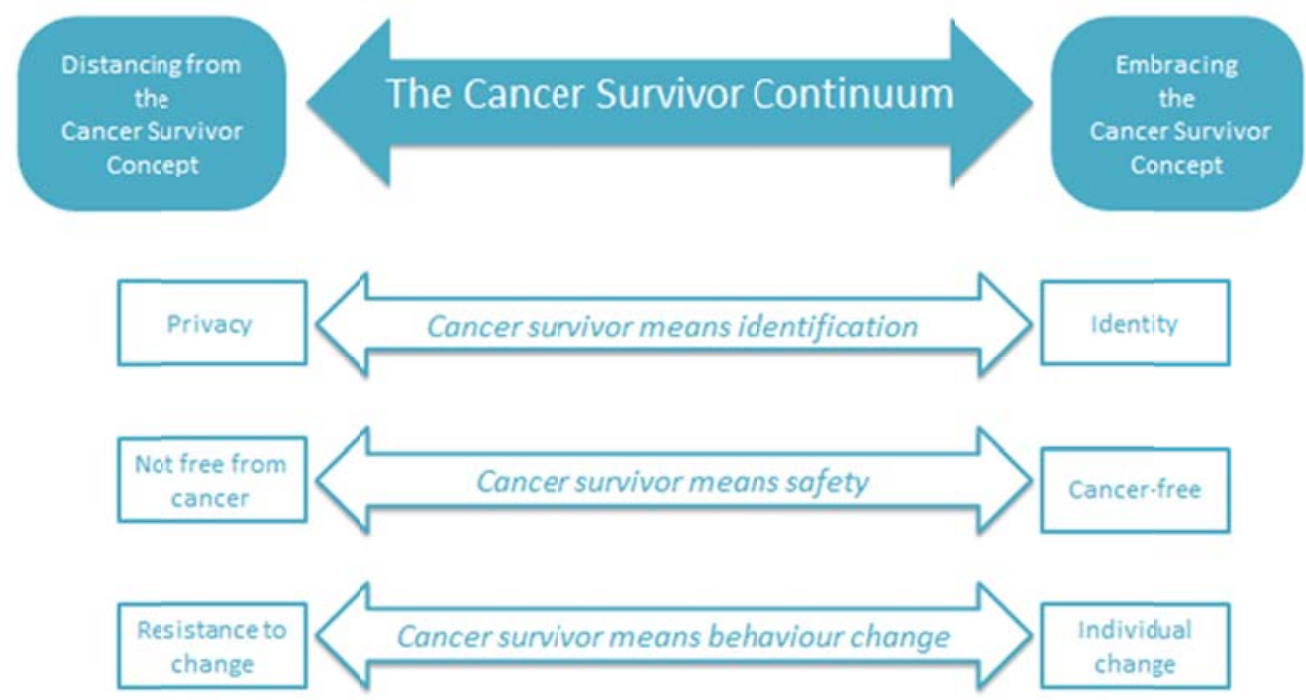

Figure 1. The Cancer Survivor Continuum

Figure 1. Each theme is represented by a continuum to illustrate there was a range of participant responses to the interview questions. The three themes represent the different meanings associated with the term cancer survivor and can be combined to form an overall continuum known as the Cancer Survivor Continuum. The Cancer Survivor Continuum represents potential reasons for embracing or distancing oneself from the cancer survivor concept. Each of the three themes and the continuums they represent are placed below the overall continuum to illustrate that they contribute to the extent that a person embraces the term cancer survivor and the meanings associated with it, or not.

\section{Discussion}

The participants of this study reported that the term cancer survivor was frequently encountered throughout their breast cancer experiences. This is in accordance with a number of authors' claims that the term cancer survivor is ubiquitous in the breast cancer culture of North America ${ }^{[5,10,12,18,19,30]}$. However, a number of authors, cancer advocacy groups, and research organizations have reported that the meaning of the term cancer survivor remains unclear ${ }^{[4,5,6,11,40]}$. Mullan ${ }^{[16]}$ has argued that the ambiguity inherent in the term cancer survivor is intentional and allows for a person to mold the term to suit his or her needs.

The results of this study indicate that women diagnosed with breast cancer associated specific meanings with the term cancer survivor. The first theme, Cancer survivor means identification, was used to explore how many of the participants used the term cancer survivor as a conventional and socially understood way of revealing their cancer histories. Data analysis also revealed that participants used the term cancer survivor to attribute deeper meanings to their experiences with cancer. These deeper meanings transformed the term beyond a mere categorization and in some cases it became a metaphor for life after cancer. This is similar to the definition of the term cancer survivor provided by previous authors ${ }^{[1,15,16,24]}$.

The second theme was developed from the data to elaborate on the connotation of safety that is inherent to the term cancer survivor. Participants discussed how they embraced the term cancer survivor because it reinforced their beliefs that they were free from cancer. This echoes what King ${ }^{[12]}$ observed while conducting ethnographic research at a Susan G. Komen Run for the Cure event where upbeat music such as "I will survive" is played throughout the festivities that celebrate life beyond breast cancer. However, the other side of this theme discussed how some participants were hesitant to use the term cancer survivor because it diminished their experiences with breast cancer and marginalized their fears concerning long-term effects of the illness. This finding is in agreement with previous research that has reported that women distance themselves from the use of the term cancer survivor because they fear long-term effects of their medical histories ${ }^{[3,23,30]}$. 
The final theme emerged from the apparent connection between the term cancer survivor and behavior change. Some participants reported that maintaining their titles as cancer survivors required them to take on specific behaviors such as advocating for healthy lifestyle decisions (e.g. routine check-ups, avoiding harmful substances, being physically active, etc.). This highlights an important aspect of the term cancer survivor and the way this term may alter the behavior of those it influences. Later in the cancer survivor means behavior change theme, results showed that some of the participants resisted the behavior changes that are associated with the cancer survivor identity. This is similar to the findings presented by Kaiser ${ }^{[12]}$ who stated that many of the participants who embraced the cancer survivor identity often resisted some of the more typical behaviors that were associated with that identity. This discussion also brings up an important point about the breast cancer culture and the culture of survivorship. There is an emphasis on individual behavior change following cancer diagnosis that perhaps broader social changes are being neglected. One example of this comes from a participant in this study. Amelia had to decide to stop dying her hair because of the potentially carcinogenic materials contained within hair dye as opposed to companies that produce hair dye having to remove those carcinogens from their products. This critique is supported by many authors who are also critical of the emphasis on individual change promoted by the cancer survivor concept $^{[10,18,29,30]}$.

This brings us to the fundamental question of this paper: do women who have been diagnosed with breast cancer embrace the term cancer survivor? Previous studies have illustrated mixed results. In her study, Kaiser ${ }^{[12]}$ reported that most participants did not embrace the term cancer survivor and rarely used this term to refer to themselves. In contrast, Document et al. ${ }^{[31]}$ stated that the vast majority of their participants embraced the term cancer survivor. The current study illustrates that perhaps the question is not so straight forward as many participants were quick to label themselves as cancer survivors but then often distanced themselves from many of the meanings associated with the term.

The meanings that the participants attributed to the term cancer survivor provided a method to determine the extent to which the women embraced the term cancer survivor or attempted to distance themselves from it. Many participants provided responses to interview questions about the term cancer survivor that indicated they had a range of ideas associated with this term.

The three themes and the range of meanings contained within them were used to develop the Cancer Survivor Continuum. The Cancer Survivor Continuum illustrates the extent to which people embrace the term cancer survivor or attempt to distance themselves from this term. A person's position along the continuum depends on the meanings that person associates with the term cancer survivor (identification, safety, or behavior change) and that person's feelings, either positive or negative, towards those meanings. As such, this continuum allows a woman to embrace certain aspects of the term cancer survivor and to distance herself from other aspects, which implies that the term cancer survivor should not be used as a general categorization. Rather, people who could be called cancer survivors may exhibit specific traits or behaviors that are typically associated with the term cancer survivor.This interpretation of the term cancer survivor allows for the possibility that a person who has been diagnosed with cancer can embrace the term and the meanings it represents. Further this interpretation allows for the possibility that a person may live beyond a cancer diagnosis without embracing the meanings that the term cancer survivor represents. This finding is in agreement with the work of Helgeson ${ }^{\text {[32] }}$. Helgeson ${ }^{[32]}$ used a questionnaire to determine the number of breast cancer related activities each of her participants $(\mathrm{N}=240)$ performed. These activities were then correlated with responses to questions regarding the extent to which the participants had integrated the breast cancer survivor identity into their self-concepts. This was done by asking questions such as "Being a breast cancer survivor is an important part of who I am" ${ }^{[32]}$. The results of Helgeson's ${ }^{[32]}$ study suggested that personal behaviors, as opposed to demographic or medical variables, more accurately predict a woman's tendency to identify herself as a breast cancer survivor.

Helgeson's ${ }^{[32]}$ results, combined with the findings of the current study, suggest that the term cancer survivor should be used on the basis of behavior as opposed to demographic or medical variables. In other words, the term cancer survivor should not be used as a general label for someone who has been diagnosed with cancer; the term cancer survivor should be 
reserved for those who feel it accurately reflects their experiences and behaviors following breast cancer diagnosis. This finding also coincides with the definition of the term cancer survivor that Mullan ${ }^{[15]}$, the person given credit for coining this term, originally intended. Mullan’s ${ }^{[15]}$ intention when creating this term was to form a description of the experiences one was likely to encounter when diagnosed with cancer and felt that survivor best captured those experiences. However, that does not necessarily mean that all people diagnosed with cancer will find the term cancer survivor and the meanings associated with it to be a suitable description of their experiences.

Using the term cancer survivor to identify a specific type of person, as opposed to all persons, living beyond a cancer diagnosis would have two effects. First, the term would be more valuable to those who chose to embrace it. As it currently stands, the term cancer survivor is not as meaningful as it could be because there are many people who are labeled with this term, but dismiss it. Secondly, those who do not embrace this term would not have to be subjected to it, and its accompanying meanings and social norms. This would allow the opportunity for development of novel, personalized, and perhaps more accurate descriptions of life after breast cancer to be created.

\subsection{Limitations and future research directions}

The main limitation of this study is that the the study team could only provide one interpretation of the data provided by the participants. The nature of human experience is extremely complex and any description of human experience could be interpreted in a number of ways. As van Manen ${ }^{[34]}$ states in his seminal work outlining the methodology of interpretive phenomenology "a phenomenological description is always one interpretation, and no single interpretation of human experience will ever exhaust the possibility of yet another complementary, or even potentially richer or deeper description ${ }^{[34]}$. Although a rigorous method for data analysis was employed throughout this study, the interpretation of the participants' lived experiences is only one interpretation and certainly should not be taken as the only valid interpretation. As such, future research is needed to corroborate the findings of this study and to add to the growing literature related to this topic. Further, additional studies could employ a form of analyst triangulation to provide diverse interpretations of the participants' data ${ }^{[36]}$. In the current study, only one researcher analyzed the data and presented interpretations of that data. Analysis by multiple researchers in future studies would strengthen the interpretations and results.

A second limitation of this study is that the examination of the lived experiences of the participants is limited to one point in time. Due to the complex nature of the issues being discussed it is possible that the participants' responses to the interview questions could be different depending on a number of factors, including mood, recent encounters with breast cancer (e.g. follow-up appointments, recent diagnosis of a family member or friend), extraneous life situations (e.g. positive/negative experiences in home-life or work-life), and stage of illness experience (e.g. time since diagnosis, ongoing secondary treatment, late-effects, etc.). As a result, future research could employ a series of interviews to determine the stability of participant responses over time and throughout their journey with breast cancer.

\subsection{Clinical implications}

Clinicians working with women who have been diagnosed with breast cancer can use the meanings those women associate with the term cancer survivor to better illicit their lived experiences with illness. For example, people who believe that the term cancer survivor conveys a sense of finality to the breast cancer experience may not like to use the term because they fear the long-term effects that their breast cancer histories may impose upon them later in life. In other words, they believe the term cancer survivor implies that the breast cancer experience is over, which is dismissive to the anxiety they feel regarding their futures. In such a case a person could be referred to services that would provide counseling to address the long-term impacts of breast cancer. Using the term cancer survivor could be a valuable tool in creating dialogue between the patient and clinician. Asking a patient: "what does the term cancer survivor mean to you?" may help elucidate many positive and negative emotions the patient is feeling and better equip the clinician to address those emotions if they are detrimental to the patient's health. 


\section{References}

[1] Bartels EC. Outside the box: Surviving survival. Literature and Medicine. 2009; 28(2): 237-252. PMid:21141796

[2] Kolata G. In one word, an entire debate on cancer. The New York Times.2004.

[3] National Coalition for Cancer Survivorship. The organization. 2011. Avaible from http://www.canceradvocacy.org/about/org/

[4] Bell K, Ristovski-Slijepcevic S. Cancer survivorship: Why labels matter. Journal of Clinical Oncology; 2013; 31(4): $409-411$. PMid:23270001 http://dx.doi.org/10.1200/JCO.2012.43.5891

[5] Khan NF, Rose PW, Evans J. Defining cancer survivorship: A more transparent approach is needed. Journal of Cancer Survivorship. 2012; 6: 33-36. PMid:21904942 http://dx.doi.org/10.1007/s11764-011-0194-6

[6] Surbone A, Annunziata MA, Santoro A, Tirelli U, Tralongo P. Cancer patients and cancer survivors: Changing words or changing culture. Annals of Oncology, Advance Access (first published online June 24, 2013). 2013.

[7] Canadian Cancer Society's Steering Committee on Cancer Statistics. Canadian Cancer Statistics 2011. Toronto, ON: Canadian Cancer Society; 2011.

[8] Jemal A, Siegel R, Xu J, Ward E. Cancer statistics, 2010. CA: a Cancer Journal for Clinicians; 2005; 60: 277-300. PMid:20610543 http://dx.doi.org/10.3322/caac.20073

[9] National Cancer Institute. 2010 Fact Book (NIH Publication No. 11-7772); 2011.

[10] King S. Pink ribbon, Inc. Minneapolis, MN: University of Minnesota Press; 2006.

[11] Twombley R. What's in a name: Who is a cancer survivor? Journal of the National Cancer Institute. 2004; 96(19): $1414-1415$. PMid:15467027 http://dx.doi.org/10.1093/jnci/96.19.1414

[12] Kaiser K. The meaning of the survivor identity for women with breast cancer. Social Science and Medicine. 2008 ; 67: 79-87. PMid:18450347 http://dx.doi.org/10.1016/j.socscimed.2008.03.036

[13] Frank AW. At the will of the body (2nd Ed.) New York: Mariner Books; 2002.

[14] Frank AW. Tricksters and truth tellers: Narrating illness in an age of authenticity and appropriation. Literature and Medicine. 2009; 28(2): 185-199. PMid:21141793

[15] Mullan F. Seasons of survival: reflections of a physician with cancer. New England Journal of Medicine. 1985; 313(4): $270-273$. PMid:4010738 http://dx.doi.org/10.1056/NEJM198507253130421

[16] Mullan F. Survivorship: an idea for everyone. In F. Mullan \& B. Hoffman (Eds.), Charting the Journey: An Almanac of Practical Resources for Cancer Survivors (pp. 1). Mount Vernon, NY: Consumer's Union; 1990. PMid:2175838

[17] Broom D. Reading breast cancer: Reflections on a dangerous intersection. Health. 2001; 5(2): 249-268.

[18] Ehrenreich B. Welcome to cancerland: a mammogram leads to a cult of pink kitsch. Harper's Magazine. 2001; 43-53.

[19] Ehrenreich B. Bright sided. New York: Henry Holt and Company; 2009.

[20] Farmer BJ, Smith ED. Breast cancer survivorship: are African American women considered? a concept analysis. Oncology Nursing Forum. 2002; 29(5): 779-787. PMid:12058153 http://dx.doi.org/10.1188/02.ONF.779-787

[21] Leopold, E. (1999). A darker ribbon. Boston, MA: Beacon Press.

[22] Lorde, A. (1980) The cancer journals. San Francisco: Aunt Lute Books

[23] Sontag S. Illness as metaphor. New York: Anchor Books; 1978.

[24] Willig C. Cancer diagnosis as discursive capture: Phenomenological repercussions of being positioned within dominant construction of cancer. Social Science \& Medicine; 2011. PMid:21440346 http://dx.doi.org/10.1016/j.socscimed.2011.02.028

[25] Kitzinger C. How to resist an idiom. Research on Language \& Social Interaction. 2000; 33(2): 121-154. http://dx.doi.org/10.1207/S15327973RLSI3302_1

[26] Klawiter M. Breast cancer in two regimes: the impact of social movements on illness experience. Sociology of Health and Illness; 2004; 26(6): 845-874. PMid:15383044 http://dx.doi.org/10.1111/j.1467-9566.2004.421_1.x

[27] Pitts V. Illness and internet empowerment: writing and reading breast cancer in cyberspace. Health. 2004; 8(1): 33-59. PMid:15018717

[28] Sharf B. Communicating breast cancer online: support and empowerment on the internet. Women \& Health. 1997; 26(1): 65-84. PMid:9311100 http://dx.doi.org/10.1300/J013v26n01_05

[29] Sinding C, Gray R. Active aging - spunky survivorship? Discourses and experiences of the years beyond breast cancer. Journal of Aging Studies. 2005; 19: 147-161. http://dx.doi.org/10.1016/j.jaging.2004.05.001

[30] Sulik GA. Pink ribbon blues: How breast cancer culture undermines women's health. New York: Oxford University Press; 2011.

[31] Document PI, Trauth JM, Key M, Flatt J, Jernigan J. Breast cancer survivors’ perception of survivorship. Oncology Nursing Forum. 2012; 39(3): 309-315. PMid:22543389 http://dx.doi.org/10.1188/12.ONF.309-315

[32] Helgeson VS. Survivor centrality among breast cancer survivors: Implications for well being. Psycho-Oncology. 2011; 20: 517-524. PMid:20878844 http://dx.doi.org/10.1002/pon.1750 
[33] Morris BA, Campbell M, Dwyer M, Dunn J, Chambers SK. Survivor identity and post-traumatic growth after participating in challenge-based peer-support programmes. British Journal of Health Psychology. 2011; 16(3): 660-674. PMid:21199541 http://dx.doi.org/10.1348/2044-8287.002004

[34] van Manen M. Researching lived experience: Human science for an action sensitive pedagogy. New York: State University of New York; 1990.

[35] Patton MQ. Qualitative research \& evaluation methods (3rd Ed.). Thousand Oaks, CA: Sage Publications, Inc.; 2002.

[36] Esterberg KG. Qualitative methods in social research. Toronto, ON: McGraw-Hill; 2002.

[37] Lincoln YS, Guba EG. Naturalistic inquiry. Newbury Park, CA: Sage Publications, Inc.; 1985.

[38] Moustakas C. Phenomenological research methods. Thousand Oaks, Ca: Sage Publications, Inc.; 1994.

[39] Glaser BG, Strauss AL. The discovery of grounded theory: Strategies for qualitative research. Chicago, IL: Aldine; 1967.

[40] Hewitt M, Greenfield S, Stovall E. (Eds.). From Cancer Patient to Cancer Survivor: Lost in Translation. Washington, DC: The National Academies Press; 2006. 